\title{
Elaidic acid induces cell apoptosis through induction of ROS accumulation and endoplasmic reticulum stress in SH-SY5Y cells
}

\author{
WEI-WEI MA ${ }^{1}$, LEI ZHAO ${ }^{2}$, LIN-HONG YUAN ${ }^{1}$, HUAN-LING YU ${ }^{1}$, \\ HUI WANG ${ }^{1}$, XIN-YUAN GONG ${ }^{1}$, FENG WEI $^{1}$ and RONG XIAO ${ }^{1}$ \\ ${ }^{1}$ Department of Nutrition and Food Hygiene, School of Public Health, Beijing Key Laboratory of Environmental Toxicology, \\ Capital Medical University, Beijing 100069, P.R. China; ${ }^{2}$ Department of Molecular Physiology and Biophysics, \\ Holden Comprehensive Cancer Center, University of Iowa Carver College of Medicine, Iowa City, IA 52242, USA
}

Received November 8, 2016; Accepted June 26, 2017

DOI: $10.3892 / \mathrm{mmr} .2017 .7830$

\begin{abstract}
Elaidic acid, which is a major trans fatty acid, has been reported to be involved in neurotoxicity; however, the underlying molecular mechanisms underlying its neurotoxic effects remain largely unknown. Therefore, the present study aimed to investigate the potential mechanisms underlying elaidic acid-induced neuronal damage in vitro. The SH-SY5Y neuroblastoma cell line was used as a model in the present study. Following treatment of cells with various concentrations of elaidic acid or with vehicle for $24 \mathrm{~h}$, cell viability was measured using the MTT assay. Mitochondrial membrane potential (MMP) and reactive oxygen species (ROS) release were measured using flow cytometry. Cell apoptosis was measured by Annexin V-fluorescein isothiocyanate/propidium iodide double staining, and cellular redox status was determined using ELISA analysis. Furthermore, western blotting was used to detect the protein expression levels of factors associated with oxidative damage and components of the endoplasmic reticulum (ER) stress/unfolded protein response (UPR) signaling pathways. The results demonstrated that elaidic acid treatment inhibited cell viability, elevated cell apoptosis and resulted in a loss of MMP. In addition, elaidic acid induced marked alterations in cellular redox status. Treatment with high doses of elaidic acid treatment also enhanced the release of ROS, and upregulated lipid peroxide and malondialdehyde levels; however, it reduced superoxide dismutase and glutathione peroxidase activities. Furthermore, elaidic acid resulted in upregulation of nuclear factor erythroid 2-related factor 2 and downregulation of heme oxygenase 1 , which are two key antioxidative factors.
\end{abstract}

Correspondence to: Dr Rong Xiao, Department of Nutrition and Food Hygiene, School of Public Health, Beijing Key Laboratory of Environmental Toxicology, Capital Medical University, 10 Xitoutiao, You An Men, Beijing 100069, P.R. China

E-mail: xiaor1222@163.com; xiaor22@ccmu.edu.cn

Key words: elaidic acid, trans fatty acid, oxidative damage, endoplasmic reticulum stress, $\mathrm{SH}-\mathrm{SY} 5 \mathrm{Y}$ cells
Elaidic acid treatment also induced or inhibited the expression of numerous ER stress/UPR-associated molecules. It induced glucose-regulated protein 78 (GRP78) expression, whereas the expression levels of activating transcription factor 4 (ATF4) and CCAAT/enhancer-binding protein homologous protein (CHOP) were upregulated and then downregulated following treatment with various doses of elaidic acid. These results indicated that elaidic acid inhibited SH-SY5Y cell growth and induced apoptosis by enhancing oxidative stress and activating the ER stress/UPR signaling pathway and the GRP78/ATF4/CHOP pathway.

\section{Introduction}

Neurodegenerative diseases, which are generally observed in the elderly, comprise a range of chronic degenerative disorders of the central nervous system (CNS), including Alzheimer's disease (AD), Parkinson's disease (PD), Huntington's disease and amyotrophic lateral sclerosis (1). These diseases are characterized by accumulation of toxic proteins, and gradual and progressive loss of neural cells, which negatively affects the mental and physical functions of patients (1). Over the last two decades, numerous potential mechanisms for the pathogenesis of neurodegenerative diseases have been proposed, including certain genetic polymorphisms, advanced age, endocrine disorders, endoplasmic reticulum (ER) stress, oxidative stress, inflammation and chemical exposure $(1,2)$. Despite progress being made in the identification of initiating factors of neurodegenerative diseases, the causative reagents associated with neuronal degeneration remain poorly understood.

Among the proposed mechanisms, the roles of ER stress and oxidative stress in the development of neuronal degeneration are of particular interest. It has previously been suggested that increased ER stress in neurons contributes to the development of $\mathrm{AD}$, which is considered one of the most common types of neurodegenerative diseases, and related cognitive impairment (3). Volgyi et al (4) reported that formation of toxic $\beta$-amyloid $(A \beta)$, which affects the ER and disturbs neurons, occurs during the development of $\mathrm{AD}$, thus suggesting that ER dysfunction may be one of the main pathological pathways in AD. Reactive oxygen species (ROS)-induced oxidative stress has also been implicated in the pathogenesis of AD 
and PD $(5,6)$. Furthermore, ROS formation and ER stress are closely linked processes, since ROS can be produced in ER due to protein misfolding and serves an important role in ER stress-induced apoptosis (7).

Recent studies have identified dietary factors, in particular the relative intake of saturated fatty acids and unsaturated fats, as being closely associated with numerous neurodegenerative disorders $(8,9)$. Trans fatty acids (TFAs) are structurally unstable saturated fatty acids with at least one double bond in the trans configuration (10). TFAs within the human diet are predominantly obtained from industrial partial hydrogenation of vegetable oils, and from the natural TFAs present in the milk and body fat of ruminants. Elaidic acid (18:1 trans-9) is the main isomer of TFAs. The pathogenic effects of TFAs have been associated with alterations in cholesterol metabolism and in the structure of biomembranes (11). Previous studies have reported that TFAs are associated with chronic diseases, including coronary heart disease, diabetes and arteriosclerosis $(12,13)$. However, the influence of TFAs on neurodegenerative disorders remains poorly understood. Epidemiological studies have revealed inconsistent results regarding the association of TFAs with the relative risk of developing AD. Morris et al reported that high dietary intake of TFAs was linked with an elevated risk of developing AD (14). However, another study failed to establish the relationship between TFA consumption and AD (15). Grimm et al reported that TFAs increased amyloidogenic processing of amyloid precursor protein, resulting in an increased production of A $\beta$ peptides in SH-SY5Y cells (16). Therefore, the effects of dietary intake of TFAs on neurodegenerative diseases and the mechanism underlying its neurotoxicity require further study.

Morinaga et al reported that the predominant isomer of TFAs, elaidic acid, plus fructose significantly increased oxidative stress and ER stress in mice and in primarily cultured hepatocytes (17). Cassagno et al reported that mice fed a diet rich in TFAs developed increased hepatic oxidative stress and ER stress (18). Given the essential roles of ER stress and oxidative stress in the development of neurodegenerative disorders, the present study explored the effects of TFAs on oxidative damage and ER stress in neurons. The molecules and signaling pathways involved in oxidative damage and ER stress were also determined, with the aim of identifying the possible mechanism underlying elaidic acid-induced neuronal damage in vitro.

\section{Materials and methods}

Cell lines and cell culture. The SH-SY5Y human neuroblastoma cell line was obtained from Peking Union Medical Center Laboratory (Beijing, China). SH-SY5Y cells were cultured in Dulbecco's modified Eagle's medium (DMEM) supplemented with $10 \%$ fetal bovine serum (HyClone; GE Healthcare Life Science, Logan, UT, USA) at $37^{\circ} \mathrm{C}$ in an atmosphere containing $5 \% \mathrm{CO}_{2}$. Elaidic acid was purchased from Sigma-Aldrich (Merck KGaA, Darmstadt, Germany) and was dissolved in DMEM to a concentration of $1 \mathrm{M}$, finally the stock solution was diluted with DMEM to a concentration of $1,000 \mu \mathrm{M}$ and stored at $-80^{\circ} \mathrm{C}$ until further use. All experiments were conducted under treatment with various doses $(10$, $20,50,100,200,400$ or $800 \mu \mathrm{M}$ ) of elaidic acid or vehicle (DMEM) for $24 \mathrm{~h}$.
MTT test. The viability of SH-SY5Y cells was determined using the MTT assay, as previously described (19). Briefly, SH-SY5Y cells were plated at $1 \times 10^{4}$ cells/well in 96-well plates in 6 replicates, and were incubated in DMEM containing elaidic acid $(10,20,50,100,200,400$ and $800 \mu \mathrm{M})$ or DMEM alone (control) for $24 \mathrm{~h}$ at $37^{\circ} \mathrm{C}$. Subsequently, $20 \mu \mathrm{l}$ MTT solution $(5 \mathrm{mg} / \mathrm{ml})$ was added to each well and the plates were incubated for $4 \mathrm{~h}$ at $37^{\circ} \mathrm{C}$. The supernatant was discarded and $200 \mu 1$ DMSO was then added to each well. The absorbance was measured at $570 \mathrm{~nm}$ using a microplate reader. In addition, SH-SY5Y cells were treated with $50 \mu \mathrm{M}$ elaidic acid for 12,24 and $48 \mathrm{~h}$, respectively, and the viability of cells were also tested by the same methods.

Measurement of ROS. Formation of ROS in cells was detected using the Fluorometric Intracellular ROS kit (Sigma-Aldrich; Merck $\mathrm{KGaA}$ ) according to the manufacturer's protocol with a slight modification (an increase in incubation time). Briefly, the collected cells were incubated with $10 \mu \mathrm{M}$ $2^{\prime}, 7^{\prime}$-dichlorofluorescin diacetate dissolved in serum-free DMEM at $37^{\circ} \mathrm{C}$ for $20 \mathrm{~min}$. The fluorescent signal was then measured using flow cytometry (BD LSRFortessa with FACSDiva software version 7.0; BD Biosciences, Franklin Lakes, NJ) with an excitation wavelength of $488 \mathrm{~nm}$ and an emission wavelength of $530 \mathrm{~nm}$.

Measurement of mitochondrial membrane potential (MMP). Alterations in MMP were measured using the MitoScreen kit (BD Bioscience, Franklin Lakes, NJ, USA) according to the manufacturer's protocol. Briefly, the SH-SY5Y cells $\left(1 \times 10^{5}\right.$ cells $/ 35 \mathrm{~mm}$ Petri dish) were collected and incubated with $0.5 \mathrm{ml} \mathrm{JC}-1$ working solution for $15 \mathrm{~min}$ at $37^{\circ} \mathrm{C}$. Cell fluorescence was measured by flow cytometry with an excitation wavelength of $488 \mathrm{~nm}$ and an emission wavelength of $530 \mathrm{~nm}$.

Annexin V-fluorescein isothiocyanate (FITC)/propidium iodide (PI) staining for apoptosis. Cells in early and late apoptosis were quantified using an Annexin V-FITC/PI double staining assay kit (KGA107; Nanjing KeyGen Biotech Co., Ltd., Nanjing, China) as described previously (20). Cells were harvested by trypsinization and washed three times with PBS. Subsequently, $1 \times 10^{5}$ cells from each $35 \mathrm{~mm}$ Petri dish were resuspended in $500 \mu \mathrm{l}$ binding buffer and stained with $5 \mu \mathrm{l}$ Annexin $\mathrm{V}$ and $5 \mu \mathrm{l}$ PI in the dark at room temperature for $20 \mathrm{~min}$. The stained cells were immediately examined using flow cytometry with an excitation wavelength of $488 \mathrm{~nm}$ for the green fluorescence of FITC-Annexin V and $561 \mathrm{~nm}$ for the red fluorescence of PI.

Measurement of oxidative stress-related biomarkers. Cells $\left(1 \times 10^{5}\right.$ cells $/ 35 \mathrm{~mm}$ Petri dish) were washed with PBS, harvested by trypsin and resuspended in $0.5 \mathrm{ml}$ PBS. An ultrasonic cell disruption system was used and the cells ruptured with ultrasonic waves for $5 \mathrm{sec}$ twice, with a $5 \mathrm{sec}$ interval, at $37^{\circ} \mathrm{C}$. Protein concentrations of the cell lysates were measured using the bicinchoninic acid (BCA) protein assay kit (Pierce; Thermo Fisher Scientific, Inc., Waltham, MA, USA). Subsequently, the levels of lipid peroxide (LPO; A106) and malondialdehyde (MDA; A003-3), the activities of superoxide dismutase (SOD; A001-3) and glutathione peroxidase (GSH-Px; A005), 
and reduced glutathione (GSH)/oxidized glutathione (GSSG) ratio (A061-1) were measured using corresponding assay kits (Nanjing Jiancheng Bioengineering Institute, Nanjing, China) according to the manufacturer's protocols.

Western blot analysis. Cells were harvested using trypsin and cell pellets were lysed in radioimmunoprecipitation assay buffer (Beijing Solarbio Science \& Technology Co., Ltd., Beijing, China) and phenylmethylsulfonyl fluoride with vigorous agitation for $15 \mathrm{~min}$ at $4^{\circ} \mathrm{C}$. The lysates were then centrifuged at $16,000 \mathrm{x} \mathrm{g}$ for $15 \mathrm{~min}$ at $4^{\circ} \mathrm{C}$ and the protein-containing supernatant was collected. Total protein concentration was determined using the BCA assay. Western blotting was performed as previously described (21). Briefly, $40 \mu \mathrm{g}$ cell lysate was separated by $12 \%$ SDS-PAGE and the proteins were transferred onto an activated polyvinylidene fluoride membrane at $60 \mathrm{~V}$ for $2 \mathrm{~h}$. The membrane was blocked with $5 \%$ non-fat dry milk in TBS containing 20\% Tween (TBST) at room temperature for $1 \mathrm{~h}$, and was then incubated with anti- $\beta$-actin, anti-nuclear factor erythroid 2-related factor 2 (Nrf2; ab31163, Abcam, Cambridge, MA, USA), anti-heme oxygenase (HO)-1 (ab13248; Abcam), anti-inositol requiring enzyme (IRE)1 $\alpha$ (9956; 1:1,000; Cell Signaling Technology, Inc., Danvers, MA, USA), anti-glucose-regulated protein (GRP)78 (9956; 1:1,000; Cell Signaling Technology, Inc.), anti-CCAAT/enhancer-binding protein homologous protein (CHOP; 9956; 1:1,000; Cell Signaling Technology, Inc.), anti-activating transcription factor (ATF) 4 (11815; 1:1,000; Cell Signaling Technology, Inc.), anti-protein disulfide isomerase (PDI; 9956; 1:1,000; Cell Signaling Technology, Inc.) and anti-endoplasmic oxidoreductin-1-like protein (Ero1-L $\alpha$; 9956; 1:1,000; Cell Signaling Technology, Inc.) primary antibodies overnight at $4^{\circ} \mathrm{C}$. Subsequently, the membrane was washed three times with TBST and was incubated with the corresponding secondary antibodies (Anti-mouse IgG, HRP-linked Antibody 7076 and Anti-rabbit IgG, HRP-linked Antibody 7074; 1:2,000; Cell Signaling Technology, Inc.) for $1 \mathrm{~h}$ at room temperature. Electrochemical luminescence (W101B; Promega Corporation, Madison, WI, USA) was used to visualize the target proteins. Images were obtained using the FluoChem ${ }^{\circledR} \mathrm{FC} 2$ imaging system (ProteinSimple; Bio-Techne, Minneapolis, MN, USA) and semi-quantification was performed by analyzing the gray values of protein bands.

Statistical analysis. All data are presented as the mean \pm standard error of at least three independent experiments. All data analyses were performed using SPSS software version 16.0 (SPSS, Inc., Chicago, IL, USA). One-way analysis of variance followed by least significant difference or Dunnett's T3 post-hoc tests were used to compare the means of different groups according to the homogeneity of variance. $\mathrm{P}<0.05$ was considered to indicate a statistically significant difference.

\section{Results}

Treatment with elaidic acid decreases cell viability. To investigate the effects of elaidic acid on cell viability, SH-SY5Y cells were treated with various concentrations of elaidic acid or vehicle for $24 \mathrm{~h}$ and cell viability was measured using the MTT assay. As presented in Fig. 1A, the viability of SH-SY5Y
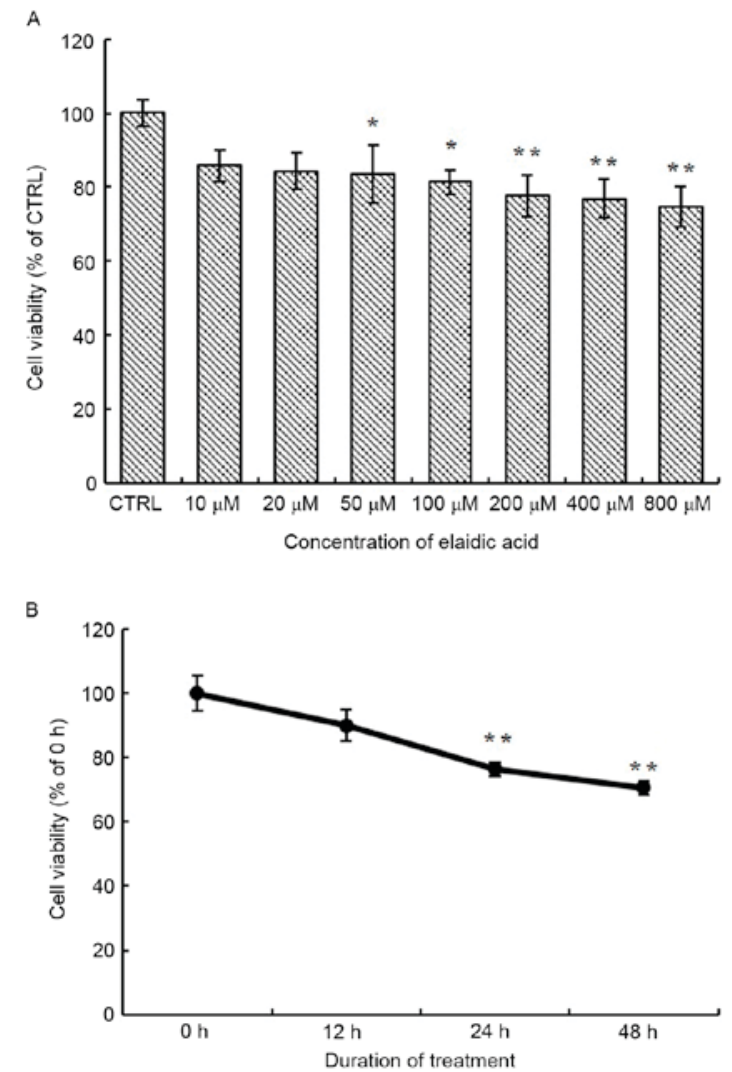

Figure 1. Treatment with elaidic acid decreases cell viability. SH-SY5Y cells were plated into a 96 -well plate at $1 \times 10^{4}$ cells/well in 6 replicates. (A) Cells were treated with DMEM alone or DMEM supplemented with elaidic acid $(10,20,50,100,200,400$ and $800 \mu \mathrm{M})$ for $24 \mathrm{~h}$. MTT assay was performed to measure cell viability. The results for each treatment were normalized to the control group. (B) Cells were treated with $50 \mu \mathrm{M}$ elaidic acid for 12, 24 and $48 \mathrm{~h}$. MTT assay was performed to measure cell viability. The results for each treatment were normalized to the $0 \mathrm{~h}$ treatment group. Data are presented as the mean \pm standard error of six replicates. ${ }^{*} \mathrm{P}<0.05,{ }^{* *} \mathrm{P}<0.01$ vs. control or $0 \mathrm{~h}$ treatment group. DMEM, Dulbecco's modified Eagle's medium.

cells was significantly reduced following treatment with 50-800 $\mu \mathrm{M}$ elaidic acid compared with the control group $(\mathrm{P}=0.044,0.022,0.007,0.005$ and 0.002 , respectively). In addition, SH-SY5Y cells were treated with $50 \mu \mathrm{M}$ elaidic acid for 12, 24 and $48 \mathrm{~h}$. Cell viability was gradually decreased as the duration of treatment was prolonged (Fig. 1B), thus suggesting that elaidic acid induced the inhibition of cell viability in a time-dependent manner.

Elaidic acid reduces MMP in SH-SY5Y cells. A distinctive feature of the early stages of apoptosis is the disruption of active mitochondria, including alterations in the MMP and the oxidation-reduction potential of mitochondria. Therefore, the present study investigated the effects of elaidic acid on MMP. The results indicated that the ratio of green fluorescence to red fluorescence was significantly elevated in cells treated with 20-800 $\mu \mathrm{M}$ elaidic acid $(\mathrm{P}<0.01)$, indicating the loss of MMP and the occurrence of early apoptotic events. In addition, the extent of the MMP was increased as the concentration of elaidic acid increased (Fig. 2A and B). These findings indicated that elaidic acid induced early apoptosis of SH-SY5Y cells in a dose-dependent manner. 

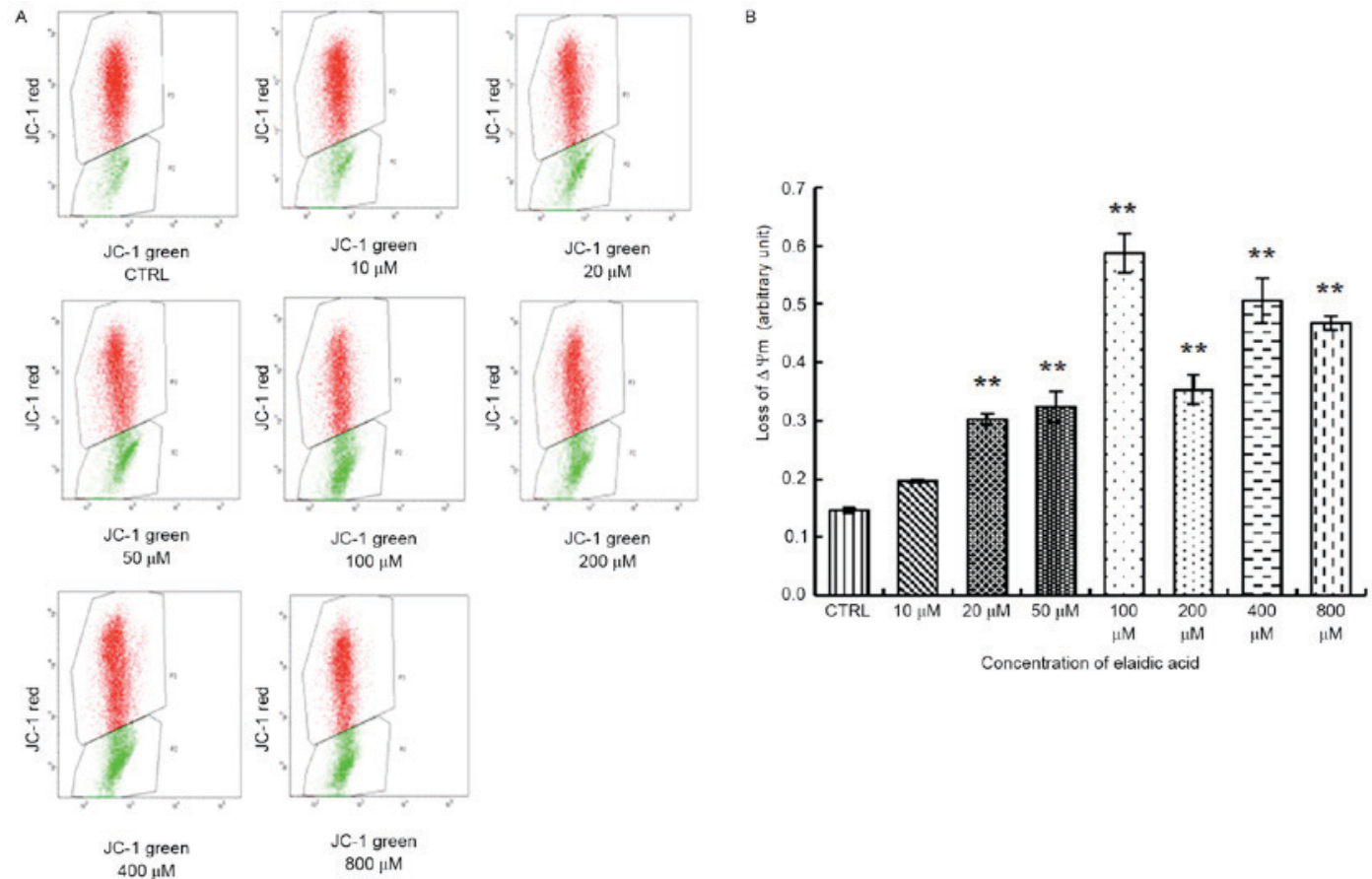

Figure 2. Treatment with elaidic acid leads to loss of MMP in SH-SY5Y cells. SH-SY5Y cell were treated with Dulbecco's modified Eagle's medium or elaidic acid $(10,20,50,100,200,400$ and $800 \mu \mathrm{M}$ ) for $24 \mathrm{~h}$. MMP was measured by JC-1 staining and flow cytometry. (A) Representative dot plots. Red fluorescence $(488 \mathrm{~nm})$ reflects the aggregated form of JC-1, whereas green fluorescence $(530 \mathrm{~nm}$ ) reflects collapsed MMP. (B) Quantification of the ratio of green signal to red signal. Data are presented as the mean \pm standard error of three independent experiments. ${ }^{* *} \mathrm{P}<0.01 \mathrm{vs}$. control group. MMP, mitochondrial membrane potential.
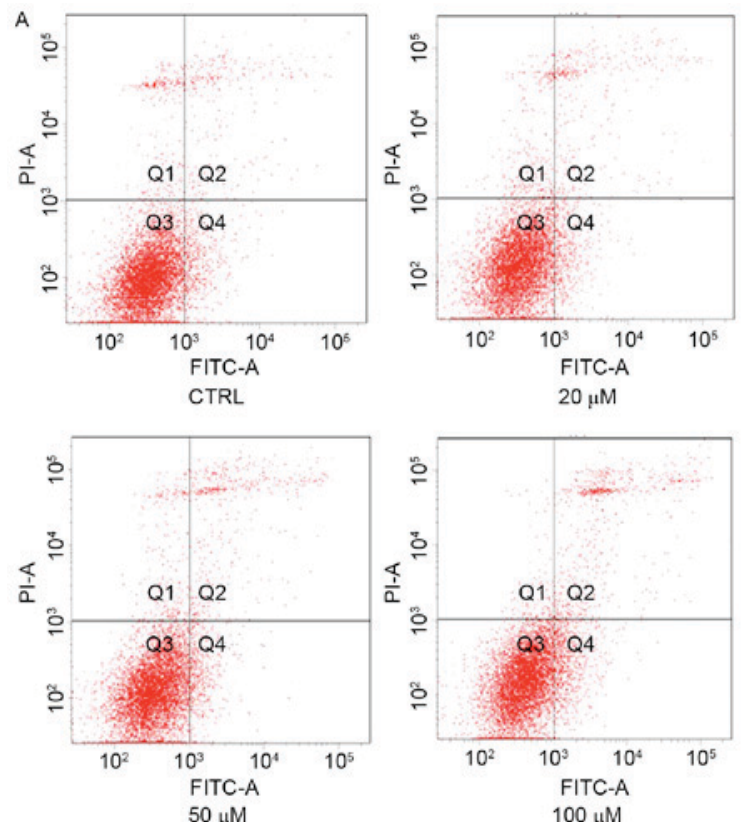

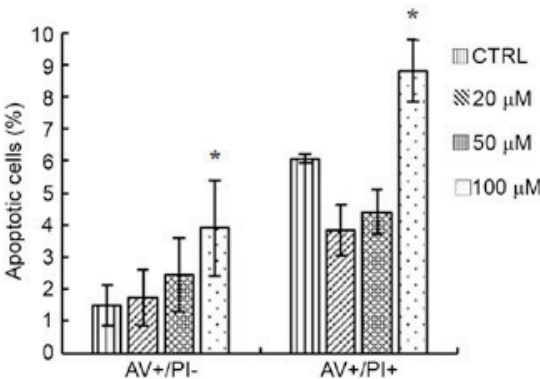

Figure 3. Elaidic acid induces apoptosis of SH-SY5Y cells. Cells were treated with Dulbecco's modified Eagle's medium or elaidic acid (20, 50 and $100 \mu \mathrm{M})$ for 24 h. Cell apoptosis was measured using AV-FITC/PI double staining and flow cytometry. (A) Representative dot plots. (B) Quantification of flow cytometry results. Data are presented as the mean \pm standard error of three independent experiments. *P<0.05 vs. control group. AV, Annexin V; FITC, fluorescein isothiocyanate; PI, propidium iodide.

Elaidic acid induces SH-SY5Y cell apoptosis. The present study detected the apoptosis of SH-SY5Y cells following treatment with 20,50 and $100 \mu \mathrm{M}$ elaidic acid using Annexin V-FITC/PI double staining and flow cytometry. As presented in Fig. 3A and B, compared with the control group, cells treated with $100 \mu \mathrm{M}$ elaidic acid exhibited a significantly higher percentage of apoptotic cells.

Elaidic acid induces elevated ROS release from $\mathrm{SH}$-SY5Y cells. Previous studies have indicated that elaidic acid may 
Table I. Effects of EA on MDA and LPO levels in SH-SY5Y cells.

\begin{tabular}{|c|c|c|c|c|}
\hline $\begin{array}{l}\text { EA concentration } \\
(\mu \mathrm{M})\end{array}$ & $\begin{array}{l}\text { MDA levels } \\
\text { (nmol/mg prot) }\end{array}$ & P-value & $\begin{array}{c}\text { LPO levels } \\
\text { (nmol/mg prot) }\end{array}$ & P-value ${ }^{a}$ \\
\hline Control & $0.38 \pm 0.02$ & - & $4.33 \pm 0.36$ & - \\
\hline 10 & $1.06 \pm 0.20$ & 0.230 & $5.65 \pm 0.15$ & 0.072 \\
\hline 20 & $1.03 \pm 0.27$ & 0.513 & $5.74 \pm 0.34$ & 0.056 \\
\hline 50 & $3.39 \pm 0.73$ & 0.143 & $5.87 \pm 0.22$ & 0.038 \\
\hline 100 & $3.50 \pm 0.26$ & 0.003 & $6.25 \pm 0.45$ & 0.011 \\
\hline 200 & $5.91 \pm 0.63$ & 0.010 & $6.12 \pm 0.84$ & 0.017 \\
\hline 400 & $2.46 \pm 0.14$ & 0.001 & $5.89 \pm 0.53$ & 0.035 \\
\hline 800 & $4.57 \pm 0.21$ & 0.013 & $8.04 \pm 0.67$ & $<0.001$ \\
\hline
\end{tabular}

${ }^{\mathrm{a}} \mathrm{VS}$. control. MDA data are presented as the mean \pm standard error of five independent experiments. LPO data are presented as the mean \pm standard error of four independent experiments. EA, elaidic acid; LPO, lipid peroxide; MDA, malondialdehyde.
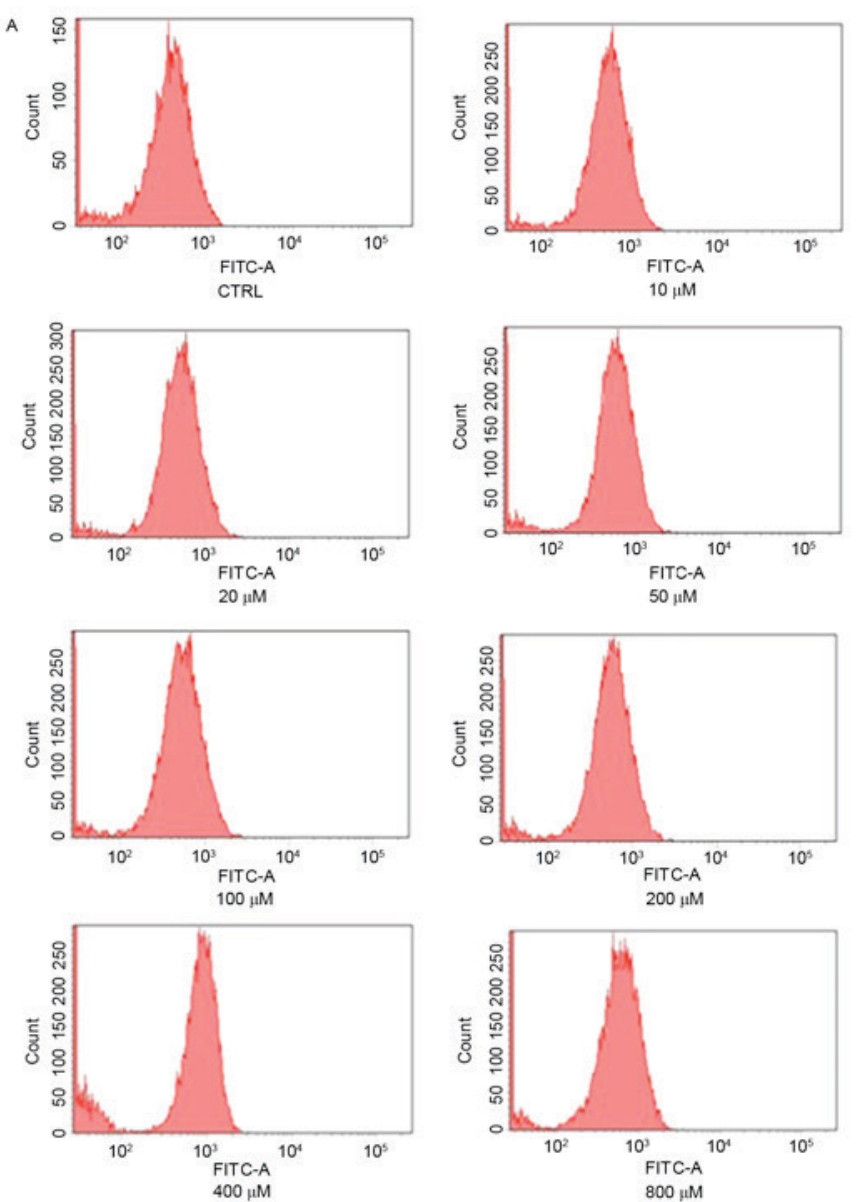

B

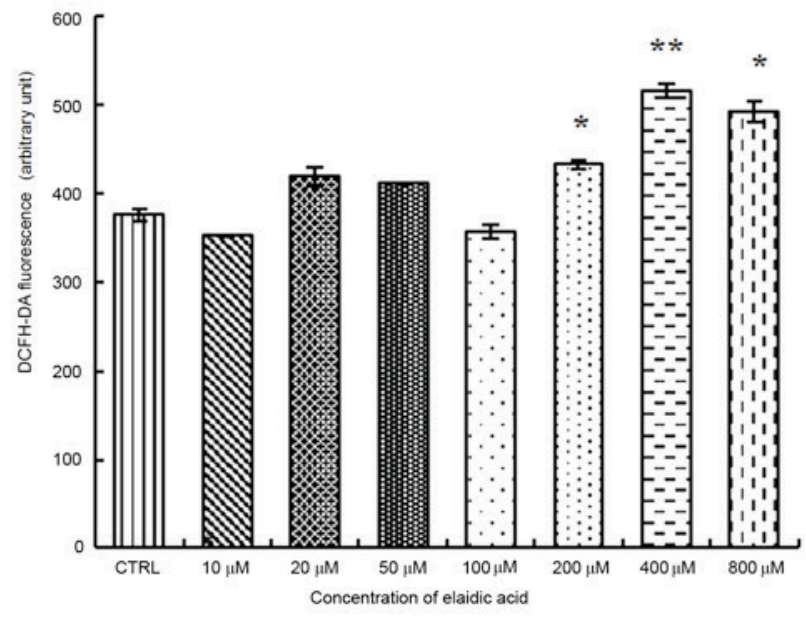

Figure 4. Elaidic acid induces elevated ROS release from SH-SY5Y cells. Cells were treated with Dulbecco's modified Eagle's medium or elaidic acid (10, 20, $50,100,200,400$ and $800 \mu \mathrm{M}$ ) for $24 \mathrm{~h}$. (A) Production of intracellular ROS was examined using a fluorometric ROS detection kit. The fluorescent signal was then measured using flow cytometry with an excitation wavelength of $488 \mathrm{~nm}$ and an emission wavelength of $530 \mathrm{~nm}$. (B) Quantification of flow cytometry results. Data are presented as the mean \pm standard error of three replicates. ${ }^{*} \mathrm{P}<0.05,{ }^{* * *} \mathrm{P}<0.01$ vs. control group. DCFH-DA, $2^{\prime}, 7^{\prime}-$ dichlorofluorescin diacetate; FITC, fluorescein isothiocyanate; ROS, reactive oxygen species.

induce oxidative stress, which is an important trigger of cell apoptosis, in liver cells $(17,18)$. Therefore, the present study examined whether elaidic acid was able to promote the generation of ROS, which is an inducer of oxidative stress in SH-SY5Y cells. As presented in Fig. 4, increased ROS release was observed in cells treated with 200,400 or $800 \mu \mathrm{M}$ elaidic acid $(\mathrm{P}=0.044,0.002$ and 0.019 , respectively).

Effects of elaidic acid on cellular redox indicators. The MDA levels in cells treated with 100-800 $\mu \mathrm{M}$ elaidic acid 
Table II. Effects of EA on SOD activity and GSH-Px levels.

\begin{tabular}{|c|c|c|c|c|}
\hline $\begin{array}{l}\text { EA concentration } \\
(\mu \mathrm{M})\end{array}$ & SOD activity (\%) & P-value ${ }^{a}$ & $\begin{array}{l}\text { GSH-Px levels } \\
\text { (U/mg prot) }\end{array}$ & P-value ${ }^{a}$ \\
\hline Control & $50.0 \pm 1.62$ & - & $39.74 \pm 7.87$ & - \\
\hline 10 & $41.1 \pm 2.35$ & 0.203 & $24.43 \pm 4.39$ & 0.066 \\
\hline 20 & $40.3 \pm 1.93$ & 0.073 & $45.33 \pm 6.31$ & 0.491 \\
\hline 50 & $37.0 \pm 3.01$ & 0.093 & $40.42 \pm 3.98$ & 0.934 \\
\hline 100 & $49.1 \pm 5.23$ & 1.000 & $32.65 \pm 4.07$ & 0.356 \\
\hline 200 & $48.5 \pm 5.13$ & 1.000 & $47.48 \pm 4.52$ & 0.314 \\
\hline 400 & $37.6 \pm 5.45$ & 0.607 & $23.03 \pm 4.80$ & 0.028 \\
\hline 800 & $26.8 \pm 1.43$ & $<0.001$ & $23.08 \pm 5.99$ & 0.035 \\
\hline
\end{tabular}

Data are presented as the mean \pm standard error of six independent experiments. ${ }^{\mathrm{a}} \mathrm{vs}$. control. EA, elaidic acid; GSH-Px, glutathione peroxidase; SOD, superoxide dismutase.

Table III. Effects of EA on GSH/GSSG ratio.

\begin{tabular}{|c|c|c|c|c|c|c|}
\hline $\begin{array}{l}\text { EA concentration } \\
(\mu \mathrm{M})\end{array}$ & $\mathrm{GSSG}(\mu \mathrm{mol} / \mathrm{l})$ & P-value ${ }^{a}$ & $\mathrm{GSH}(\mu \mathrm{mol} / \mathrm{l})$ & P-value ${ }^{a}$ & GSH/GSSG ratio & P-value ${ }^{a}$ \\
\hline Control & $9.84 \pm 0.94$ & - & $34.24 \pm 2.24$ & - & $3.68 \pm 0.58$ & - \\
\hline 10 & $10.40 \pm 0.56$ & 0.502 & $36.78 \pm 2.68$ & 0.520 & $3.62 \pm 0.43$ & 1.000 \\
\hline 20 & $9.29 \pm 0.38$ & 0.502 & $50.59 \pm 3.29$ & $<0.001$ & $5.50 \pm 0.47$ & 0.461 \\
\hline 50 & $9.79 \pm 0.20$ & 0.949 & $40.89 \pm 3.05$ & 0.098 & $4.21 \pm 0.39$ & 1.000 \\
\hline 100 & $10.46 \pm 0.54$ & 0.453 & $37.21 \pm 3.24$ & 0.452 & $3.55 \pm 0.20$ & 1.000 \\
\hline 200 & $11.78 \pm 0.38$ & 0.024 & $29.33 \pm 1.77$ & 0.217 & $2.49 \pm 0.11$ & 0.688 \\
\hline 400 & $11.37 \pm 0.66$ & 0.070 & $33.14 \pm 2.12$ & 0.779 & $2.99 \pm 0.34$ & 0.997 \\
\hline 800 & $10.87 \pm 0.65$ & 0.216 & $23.96 \pm 3.21$ & 0.013 & $2.28 \pm 0.42$ & 0.730 \\
\hline
\end{tabular}

Data are presented as the mean \pm standard error of five independent experiments. ${ }^{\mathrm{a}} \mathrm{vs}$. control. EA, elaidic acid; GSH, reduced glutathione; GSSG, oxidized glutathione.

were significantly upregulated compared with in the control group (Table I). LPO levels were also increased following treatment with 50-800 $\mu \mathrm{M}$ elaidic acid (Table I). Elaidic acid only affected SOD activity in the $800 \mu \mathrm{M}$ treatment group, in which SOD activity was significantly reduced $(\mathrm{P}<0.05$; Table II). In addition, 400 and $800 \mu \mathrm{M}$ elaidic acid decreased GSH-Px activity in SH-SY5Y cells ( $\mathrm{P}<0.05$; Table II). In addition, decreased GSH levels were detected in the $800 \mu \mathrm{M}$ elaidic acid group, whereas the levels of GSSG were increased in the $200 \mu \mathrm{M}$ elaidic acid group $(\mathrm{P}<0.05)$. However, the ratio of $\mathrm{GSH}$ and GSSG showed no significant difference among the groups (P>0.05; Table III).

Effects of elaidic acid on expression of antioxidative factors. Nrf2 regulates the expression of a series of antioxidant proteins, including $\mathrm{HO}-1$, which protect cells against oxidative damage (22). In response to oxidative stress, Nrf2 and HO-1 expression may be upregulated (23). Therefore, the present study measured the expression levels of Nrf2 and HO-1 in response to elaidic acid-induced ROS accumulation. As presented in Fig. 5A and B, the protein expression levels of Nrf2 were upregulated following treatment with 200, 400 and $800 \mu \mathrm{M}$ elaidic acid at $(\mathrm{P}=0.008,0.008$ and 0.002 , respectively), whereas the expression of $\mathrm{HO}-1$ was significantly inhibited by elaidic acid at 400 and $800 \mu \mathrm{M}(\mathrm{P}=0.013$ and 0.001 , respectively). Although HO-1 protein expression appeared increased following treatment with elaidic acid between 10 and $200 \mu \mathrm{M}$, no statistical significance was detected. These results suggested that other mechanisms may participate in the regulation of HO-1 expression, and the loss of HO-1-induced protection following treatment with high concentrations of elaidic acid may contribute to elaidic acid-induced apoptosis.

Elaidic acid induces ER stress in SH-SY5Y cells. Production of ROS is known to be associated with ER stress and activation of the UPR. Overactivation of the UPR has been reported to induce apoptotic cell death and contribute to various degenerative diseases. In the present study, the protein expression levels of GRP78 were significantly upregulated following treatment with 400 and $800 \mu \mathrm{M}$ elaidic acid $(\mathrm{P}=0.019$ and 0.007 , respectively; Fig. 6A and B). ATF4 protein expression was upregulated following treatment with $20-400 \mu \mathrm{M}$ $(\mathrm{P}=0.019,0.001,<0.001,0.002$ and 0.043 , respectively; Fig. 6A and B). However, elaidic acid downregulated CHOP 
expression at the concentrations of 200,400 and $800 \mu \mathrm{M}$ $(\mathrm{P}=0.028,0.016$ and 0.011 , respectively; Fig. 6A and $\mathrm{B})$. In addition, no significant alterations were detected in the protein expression levels of IRE1 $\alpha$ following treatment with elaidic acid ( $\mathrm{P}>0.05$; Fig. 6A and B). During ER stress, disulfide bond formation is dysregulated, since disulfide bond formation in the ER lumen is highly sensitive to altered redox balance, which in turn exacerbates ROS accumulation and oxidative stress. However, the present study failed to detect significant alterations in the protein expression levels of PDI and Ero1-L $\alpha$, which are two major proteins associated with disulfide bond formation, in cells treated with elaidic acid ( $\mathrm{P}>0.05$; Fig. 6C and D).

\section{Discussion}

In the present study, elaidic acid between 10 and $800 \mu \mathrm{M}$ was selected to investigate its neurotoxic effects in SH-SY5Y cells in vitro, according to the physiological dose of elaidic acid, which is between 10 and $80 \mu \mathrm{M}$ (24-29). The results demonstrated that the cell viability was decreased following treatment with elaidic acid in a dose- and time-dependent manner, indicating the neurotoxic effects of elaidic acid. Destruction of MMP is considered an early event in cell apoptosis. The results of the present study demonstrated that various doses of elaidic acid were able to decrease MMP of cells in a dose-dependent manner, indicating that elaidic acid-induced loss of SH-SY5Y cell viability was at least partially attributed to apoptosis. Annexin V/PI double staining also confirmed that $100 \mu \mathrm{M}$ elaidic acid induced cell apoptosis. Taken together, these data indicated that elaidic acid could induce growth inhibition and apoptosis of SH-SY5Y neuroblastoma cells, thus suggesting a potential role for elaidic acid in the development of neuronal loss in neurodegenerative diseases.

Morinaga et al (17) and Cassagno et al (18) reported that elaidic acid caused oxidative stress and ER stress in mouse hepatocytes and liver tissues. Neurons are particularly vulnerable to oxidative stress due to their high oxygen consumption. Oxidative stress is known to serve an essential role in the pathogenesis of neurodegenerative disorders. ROS can be generated in the mitochondria, ER, plasma membrane and cytoplasm, and induces oxidative stress and ER stress. The present study demonstrated that, at high concentrations, elaidic acid enhanced ROS release, which may lead to cell oxidative damage and ultimately cell apoptosis.

To limit overaccumulation of ROS in the body, enzymatic and non-enzymatic systems exist to maintain ROS balance. Enzymatic antioxidant defenses include SOD and GSH-Px. GSH in the nucleus maintains the redox state of sulfhydryls of critical proteins for DNA repair and gene expression. Under oxidative stress, GSH-Px is a peroxide decomposition enzyme and has a specific catalytic role in the oxide reduction reaction of GSH, whose functions are eliminating peroxide metabolites and protecting cell membrane structure and function. SOD converts ROS to $\mathrm{H}_{2} \mathrm{O}_{2}$; therefore, SOD possesses the ability to act as a free radical scavenging enzyme. Lipid peroxidation of unsaturated fatty acids in the cell membrane, which is triggered by free radicals, results in the formation of LPO; therefore, LPO content may reflect free radical content and lipid peroxidation in cells. A reduction in SOD

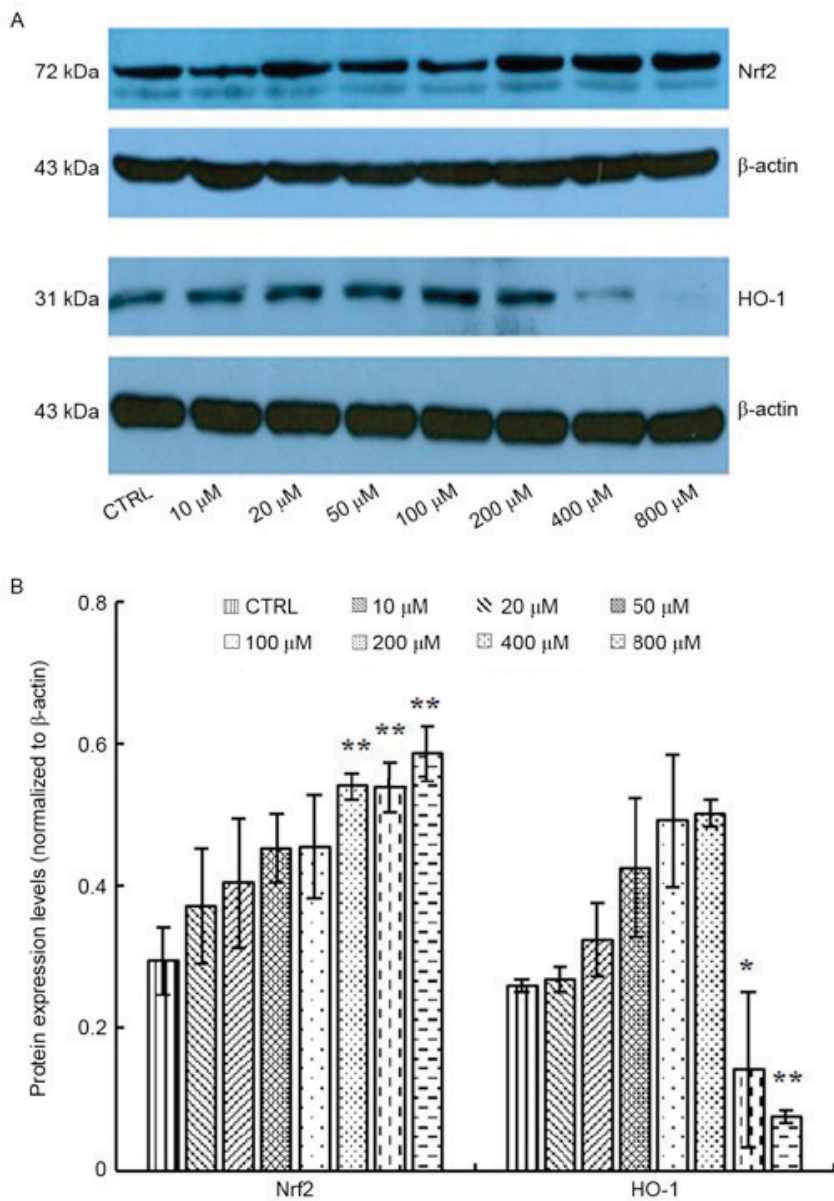

Figure 5. Effects of elaidic acid on the expression levels of antioxidative factors. Cells were treated with Dulbecco's modified Eagle's medium or elaidic acid $(10,20,50,100,200,400$ and $800 \mu \mathrm{M})$ for $24 \mathrm{~h}$. The protein expression levels of $\mathrm{Nrf} 2$ and HO-1 were detected by western blotting. (A) Representative blotting image. (B) Semi-quantification of the western blotting data. Data are presented as the mean \pm standard error of three independent experiments. ${ }^{*} \mathrm{P}<0.05,{ }^{* *} \mathrm{P}<0.01$ vs. control group. HO-1, heme oxygenase-1; Nrf2, nuclear factor erythroid 2-related factor 2.

activity, which in turn may trigger the breakdown of LPO to MDA, indicates cell toxicity. The results of the present study demonstrated that, compared with the control group, cells treated with $800 \mu \mathrm{M}$ elaidic acid exhibited decreased GSH content and SOD and GSH-Px activities, whereas LPO and MDA levels were increased. These results indicated that elaidic acid impaired the ability of SH-SY5Y cells to scavenge ROS and in turn resulted in the formation of LPO and its metabolic product, MDA, thus suggesting the existence of oxidative damage in cells. In addition, elaidic acid caused a decrease in GSH-Px, which contributed to GSSG formation by GSH; therefore, reduced GSH but increased GSSG levels were detected.

Under oxidative stress, $\mathrm{Nrf} 2$ translocates into the nucleus of cells, where it binds with the antioxidant response element (30). HO-1 protein expression, which is regulated by Nrf2 (22), may be enhanced in response to oxidative stress (23). In the present study, upregulation of $\mathrm{Nrf} 2$ and $\mathrm{HO}-1$ were detected in response to low doses of elaidic acid, which indicated that Nrf2 and HO-1 exerted protective effects against ROS accumulation induced by low concentrations of elaidic acid. However, when SH-SY5Y cells were treated with high concentrations 

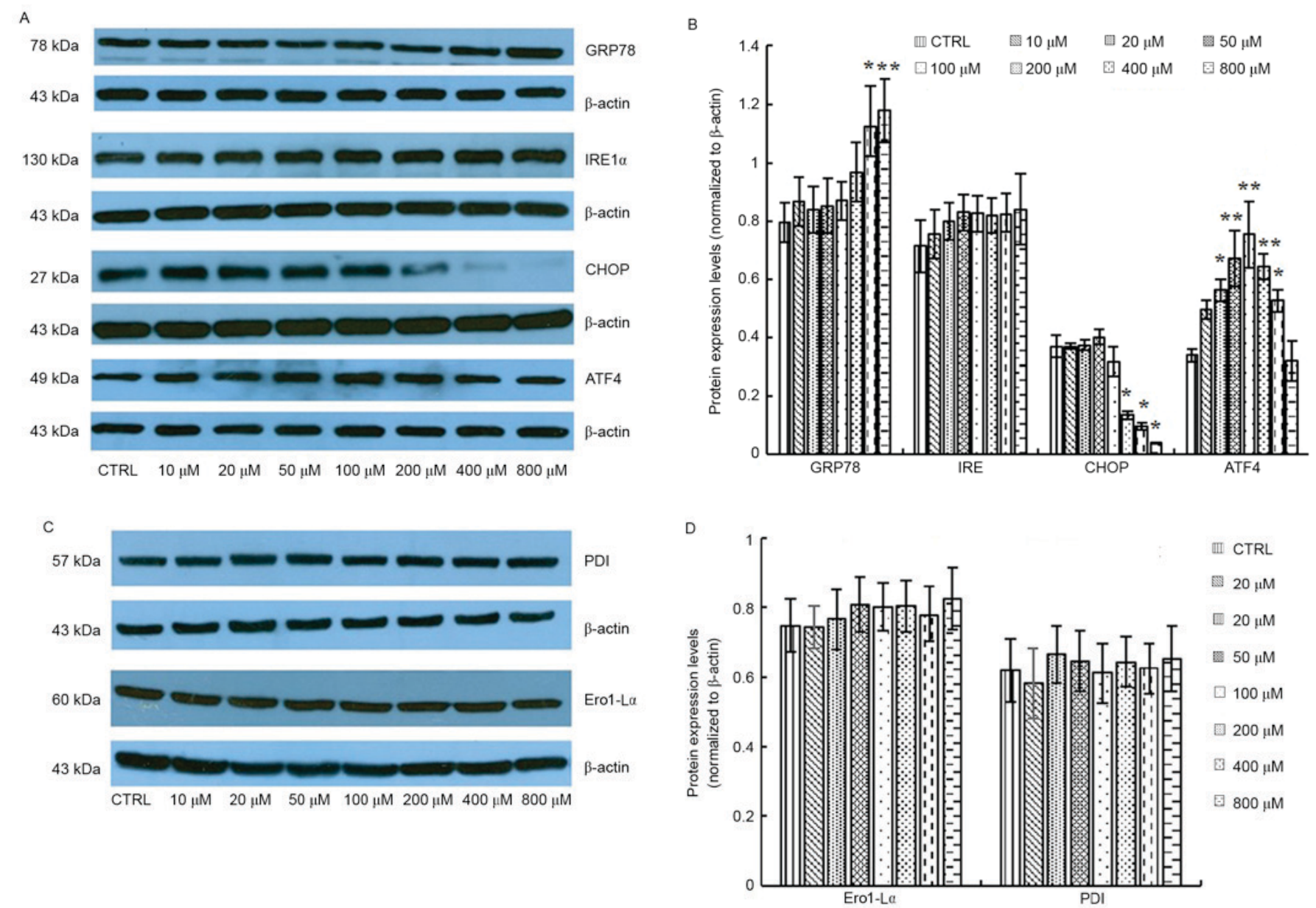

Figure 6. Elaidic acid induces endoplasmic reticulum stress in SH-SY5Y cells. Cell were treated with Dulbecco's modified Eagle's medium or elaidic acid $(10,20,50,100,200,400$ and $800 \mu \mathrm{M})$ for $24 \mathrm{~h}$. (A and B) Protein expression levels of GRP78, IRE1 $\alpha$, CHOP and ATF4 were detected by western blotting. (A) Representative blotting image. (B) Semi-quantification of the western blotting data. (C and D) Protein expression levels of PDI and Ero1-L $\alpha$ were detected by western blotting. (C) Representative blotting image. (D) Semi-quantification of the western blotting data. Data are presented as the mean \pm standard error of five independent experiments. ${ }^{*} \mathrm{P}<0.05,{ }^{* *} \mathrm{P}<0.01$ vs. control group. ATF4, activating transcription factor 4; CHOP, CCAAT/enhancer-binding protein homologous protein; Ero1-L $\alpha$, endoplasmic oxidoreductin-1-like protein; GRP78, glucose-regulated protein 78; IRE1 $\alpha$, inositol requiring enzyme 1 $\alpha$; PDI, protein disulfide isomerase.

of elaidic acid, HO-1 expression was downregulated, whereas Nrf2 expression was further upregulated, indicating that other mechanisms superseded the regulation of HO-1 by Nrf2 and that the toxic effects of high doses of elaidic acid exhausted the protective capacity of HO-1.

ER serves a pivotal role in the synthesis, folding, post-translational modifications and trafficking of secretory and membrane proteins, calcium storage and release, lipid biogenesis and apoptosis $(31,32)$. Physiological and pathological stimuli may lead to disruption of ER homeostasis and result in an accumulation of misfolded and unfolded proteins; this condition is known as ER stress. ER stress activates three main signaling pathways (ATF6, IRE1 and PERK) to reduce ER stress and restore homeostasis, which is referred to as the UPR. However, if ER stress is excessive and prolonged, these adaptive responses fail to compensate and overactivation of the UPR leads to cell death (33). It has previously been reported that oxidative stress-induced ER stress may be crucial in the regulation of cell apoptosis and may contribute to various degenerative diseases (34). ROS production has been associated with ER stress and activation of the UPR. In addition, some UPR components, such as CHOP, can contribute to oxidative stress (35). Meanwhile, ER stress can cause mitochondrial dysfunction and increase mitochondrial ROS production. In numerous ER stress models, ER stress and oxidative stress accentuate each other in a positive feedback loop, which interferes with cell function and activates proapoptotic signaling (36).

IRE1 $\alpha$ is a highly conserved protein in neurons and the major neuronal UPR sensor $(37,38)$. Under normal physiological conditions, IRE1 $\alpha$ is activated by dimerization and trans-autophosphorylation upon its release from GRP78 $(39,40)$. The released IRE1 $\alpha$ migrates to the nucleus and induces the transcription of ER chaperone proteins, including GRP78 and CHOP (41). ATF4 is a master regulator that has a crucial role in stress adaptation via regulation of the transcription of numerous genes, including CHOP. In the present study, following elaidic acid treatment, the expression levels of GRP78 were upregulated, indicating that ER stress was induced. However, IRE1 $\alpha$ expression was not significantly altered, whereas the expression levels of CHOP and ATF4 were upregulated, and then downregulated, following treatment with various doses of elaidic acid, indicating that as the dose of elaidic was increased, the damage to cells was 
aggravated. These results suggested that the effects of elaidic acid on ER stress were mainly mediated via activation of the GRP78/ATF4/CHOP signaling pathway.

The ER redox state is closely associated with ER protein-folding homeostasis. Disulfide bond formation in the ER lumen is highly sensitive to altered redox balance, where both reducing and oxidizing reagents disrupt protein folding and cause ER stress. During ER stress, dysregulated disulfide bond formation and breakage may result in ROS accumulation and the induction of oxidative stress. Two of the major contributors to disulfide bond formation in the ER are PDI and Erol (42). However, the present study did not observe alterations in the expression of PDI and Erol in SH-SY5Y cells treated with elaidic acid, indicating that elaidic acid has no effect on the formation of disulfide bonds and protein conformation.

In conclusion, elaidic acid may induce apoptosis of SH-SY5Y cells via the induction of oxidative stress and ER stress, and through activation of the GRP78/ATF4/CHOP signaling pathway. These findings suggested a potential role for dietary TFAs in the development of neurodegenerative disorders, providing the basis for the development of novel strategies for prevention of neurodegenerative disorders. Animal-based in vivo experiments are required to confirm the conclusion of this research in further studies.

\section{Acknowledgements}

The present study was supported by grants from the National Natural Science Foundation of China (grant no. 81472982), the Support Project of High-level Teachers in Beijing Municipal Universities in the Period of 13th Five-year Plan (grant no. CIT\&TCD201704096) and the Scientific Research Common Program of Beijing Municipal Commission of Education (grant no. KM201710025007).

\section{References}

1. Forman MS, Trojanowski JQ and Lee VM: Neurodegenerative diseases: A decade of discoveries paves the way for therapeutic breakthroughs. Nat Med 10: 1055-1063, 2004.

2. Brown RC, Lockwood AH and Sonawane BR: Neurodegenerative diseases: An overview of environmental risk factors. Environ Health Perspect 113: 1250-1256, 2005.

3. Rozpedek W, Markiewicz L, Diehl JA, Pytel D and Majsterek I: Unfolded protein response and PERK kinase as a new therapeutic target in the pathogenesis of alzheimer's disease. Curr Med Chem 22: 3169-3184, 2015.

4. Volgyi K, Juhász G, Kovacs Z and Penke B: Dysfunction of endoplasmic reticulum (ER) and mitochondria (MT) in Alzheimer's disease: The role of the ER-MT cross-talk. Curr Alzheimer Res 12: 655-672, 2015.

5. Castellani R, Hirai K, Aliev G, Drew KL, Nunomura A, Takeda A, Cash AD, Obrenovich ME, Perry G and Smith MA: Role of mitochondrial dysfunction in Alzheimer's disease. J Neurosci Res 70: 357-360, 2002.

6. Blesa J, Trigo-Damas I, Quiroga-Varela A and Jackson-Lewis VR: Oxidative stress and Parkinson's disease. Front Neuroanat 9: 91, 2015.

7. Malhotra JD and Kaufman RJ: Endoplasmic reticulum stress and oxidative stress: A vicious cycle or a double-edged sword? Antioxid Redox Signal 9: 2277-2293, 2007

8. Barnard ND, Bunner AE and Agarwal U: Saturated and trans fats and dementia: A systematic review. Neurobiol Aging 35 (Suppl 2): S65-S73, 2014.

9. Morris MC and Tangney CC: Dietary fat composition and dementia risk. Neurobiol Aging 35 (Suppl 2): S59-S64, 2014.
10. Lichtenstein AH: Dietary trans fatty acids and cardiovascular disease risk: Past and present. Curr Atheroscler Rep 16: 433, 2014.

11. Vendel Nielsen L, Krogager TP, Young C, Ferreri C, Chatgilialoglu C, Nørregaard Jensen $O$ and Enghild JJ: Effects of elaidic acid on lipid metabolism in HepG2 cells, investigated by an integrated approach of lipidomics, transcriptomics and proteomics. PLoS One 8: e74283, 2013.

12. Perova NV, Metel'skaia VA and Bortsov SA: Trans isomers of unsaturated fatty acids increase the risk of atherosclerosis-related circulatory system diseases. Ter Arkh 85: 113-117, 2013. (In Russian)

13. de Souza RJ, Mente A, Maroleanu A, Cozma AI, Ha V, Kishibe T, Uleryk E, Budylowski P, Schünemann H, Beyene J and Anand SS: Intake of saturated and trans unsaturated fatty acids and risk of all cause mortality, cardiovascular disease, and type 2 diabetes: Systematic review and meta-analysis of observational studies. BMJ 351: h3978, 2015.

14. Morris MC, Evans DA, Bienias JL, Tangney CC, Bennett DA, Aggarwal N, Schneider J and Wilson RS: Dietary fats and the risk of incident Alzheimer disease. Arch Neurol 60: 194-200, 2003.

15. Engelhart MJ, Geerlings MI, Ruitenberg A, Van Swieten JC, Hofman A, Witteman JC and Breteler MM: Diet and risk of dementia: Does fat matter? The rotterdam study. Neurology 59: 1915-1921, 2002.

16. Grimm MO, Rothhaar TL, Grösgen S, Burg VK, Hundsdörfer B, Haupenthal VJ, Friess P, Kins S, Grimm HS and Hartmann T: Trans fatty acids enhance amyloidogenic processing of the Alzheimer amyloid precursor protein (APP). J Nutr Biochem 23: 1214-1223, 2012.

17. Morinaga M, Kon K, Saito H, Arai K, Kusama H, Uchiyama A, Yamashina S, Ikejima $\mathrm{K}$ and Watanabe $\mathrm{S}$ : Sodium 4-phenylbutyrate prevents murine dietary steatohepatitis caused by trans-fatty acid plus fructose. J Clin Biochem Nutr 57: 183-191, 2015.

18. Cassagno N, Palos-Pinto A, Costet P, Breilh D, Darmon M and Bérard AM: Low amounts of trans 18:1 fatty acids elevate plasma triacylglycerols but not cholesterol and alter the cellular defence to oxidative stress in mice. Br J Nutr 94: 346-352, 2005.

19. Starr TK, Scott PM, Marsh BM, Zhao L, Than BL, O'Sullivan MG, Sarver AL, Dupuy AJ, Largaespada DA and Cormier RT: A sleeping beauty transposon-mediated screen identifies murine susceptibility genes for adenomatous polyposis coli (Apc)-dependent intestinal tumorigenesis. Proc Natl Acad Sci USA 108: 5765-5770, 2011.

20. Sakitani K, Hirata Y, Hikiba Y, Hayakawa Y, Ihara S, Suzuki H, Suzuki N, Serizawa T, Kinoshita H, Sakamoto K, et al: Inhibition of autophagy exerts anti-colon cancer effects via apoptosis induced by p53 activation and ER stress. BMC Cancer 15: 795, 2015.

21. Sui C, Ma Q, Nan K, Xiao J, Suo A, Sha H and Zhao L: hSSTR2 expression and octreotide treatment reverses multidrug resistance of BxPC-3 human pancreatic cancer cells. Oncol Rep 22: 1391-1396, 2009

22. Gong X, Zhang L, Jiang R, Ye M, Yin X and Wan J: Anti-inflammatory effects of mangiferin on sepsis-induced lung injury in mice via up-regulation of heme oxygenase-1. J Nutr Biochem 24: 1173-1181, 2013.

23. Takahashi T, Shimizu H, Morimatsu H, Maeshima K, Inoue K, Akagi R, Matsumi M, Katayama $\mathrm{H}$ and Morita K: Heme Oxygenase-1 is an essential cytoprotective component in oxidative tissue injury induced by hemorrhagic shock. J Clin Biochem Nutr 44: 28-40, 2009.

24. van Poppel G: Intake of trans fatty acids in western Europe: The TRANSFAIR study. Lancet 351: 1099, 1998.

25. Jokela H, Kalela A, Lilja M, Salmi M, Lehtimäki T, Kunnas T, Teisala K, Punnonen R and Nikkari ST: Sequentially combined estradiol valerate plus levonorgestrel therapy decreases 18:1 trans-fatty acid content of plasma lipids in healthy postmenopausal women. Gynecol Endocrinol 21: 360-365, 2005.

26. Svahn JC, Feldl F, Räihä NC, Koletzko B and Axelsson IE: Different quantities and quality of fat in milk products given to young children: Effects on long chain polyunsaturated fatty acids and trans fatty acids in plasma. Acta Paediatr 91: 20-29, 2002.

27. Abraham RA, Bahl VK, Parshad R, Seenu V, Roy A, Golandaz S, Dorairaj P and Ramakrishnan L: Content of trans fatty acids in human cheek epithelium: Comparison with serum and adipose tissue. Biomed Res Int 2013: 276174, 2013. 
28. Sun Q, Ma J, Campos H, Hankinson SE, Manson JE, Stampfer MJ, Rexrode KM, Willett WC and Hu FB: A prospective study of trans fatty acids in erythrocytes and risk of coronary heart disease. Circulation 115: 1858-1865, 2007.

29. Burdge GC, Tricon S, Morgan R, Kliem KE, Childs C, Jones E, Russell JJ, Grimble RF, Williams CM, Yaqoob P and Calder PC: Incorporation of cis-9, trans-11 conjugated linoleic acid and vaccenic acid (trans-11 18:1) into plasma and leucocyte lipids in healthy men consuming dairy products naturally enriched in these fatty acids. Br J Nutr 94: 237-243, 2005.

30. Itoh K, Tong KI and Yamamoto M: Molecular mechanism activating Nrf2-Keap1 pathway in regulation of adaptive response to electrophiles. Free Radic Biol Med 36: 1208-1213, 2004.

31. Henderson MJ, Baldwin HA, Werley CA, Boccardo S, Whitaker LR, Yan X, Holt GT, Schreiter ER, Looger LL, Cohen AE, et al: A low affinity GCaMP3 variant (GCaMPer) for imaging the endoplasmic reticulum calcium store. PLoS One 10: e0139273, 2015

32. Schwarz DS and Blower MD: The endoplasmic reticulum: Structure, function and response to cellular signaling. Cell Mol Life Sci 73: 79-94, 2016.

33. Bakhshi J, Weinstein L, Poksay KS, Nishinaga B, Bredesen DE and Rao RV: Coupling endoplasmic reticulum stress to the cell death program in mouse melanoma cells: Effect of curcumin. Apoptosis 13: 904-914, 2008.

34. Xu D, Perez RE, Rezaiekhaligh MH, Bourdi M and Truog WE: Knockdown of ERp57 increases BiP/GRP78 induction and protects against hyperoxia and tunicamycin-induced apoptosis. Am J Physiol Lung Cell Mol Physiol 297: L44-L51, 2009.
35. Feng J, Chen X, Sun X, Wang F and Sun X: Expression of endoplasmic reticulum stress markers GRP78 and CHOP induced by oxidative stress in blue light-mediated damage of A2E-containing retinal pigment epithelium cells. Ophthalmic Res 52: 224-233, 2014.

36. Ferreiro E, Baldeiras I, Ferreira IL, Costa RO, Rego AC, Pereira CF and Oliveira CR: Mitochondrial- and endoplasmic reticulum-associated oxidative stress in Alzheimer's disease: From pathogenesis to biomarkers. Int J Cell Biol 2012: 735206, 2012.

37. Chen Y and Brandizzi F: AtIRE1A/AtIRE1B and AGB1 independently control two essential unfolded protein response pathways in Arabidopsis. Plant J 69: 266-277, 2012.

38. Nagashima Y, Mishiba K, Suzuki E, Shimada Y, Iwata Y and Koizumi N: Arabidopsis IRE1 catalyses unconventional splicing of bZIP60 mRNA to produce the active transcription factor. Sci Rep 1: 29, 2011.

39. Kim I, Xu W and Reed JC: Cell death and endoplasmic reticulum stress: Disease relevance and therapeutic opportunities. Nat Rev Drug Discov 7: 1013-1030, 2008.

40. Bertolotti A, Zhang Y, Hendershot LM, Harding HP and Ron D: Dynamic interaction of BiP and ER stress transducers in the unfolded-protein response. Nat Cell Biol 2: 326-332, 2000.

41. Szegezdi E, Logue SE, Gorman AM and Samali A: Mediators of endoplasmic reticulum stress-induced apoptosis. EMBO Rep 7: 880-885, 2006.

42. Benham AM, van Lith M, Sitia R and Braakman I: Ero1-PDI interactions, the response to redox flux and the implications for disulfide bond formation in the mammalian endoplasmic reticulum. Philos Trans R Soc Lond B Biol Sci 368: 20110403, 2013. 